\title{
Systematic characterization of amplitude-integrated EEG signals for monitoring the preterm brain
}

\author{
Elke Griesmaier', David Pierre Enot ${ }^{1,2}$, Miriam Bachmann'1, Vera Neubauer', Lena Hellström-Westas ${ }^{3}$, \\ Ursula Kiechl-Kohlendorfer ${ }^{1}$ and Matthias Keller ${ }^{1,2}$
}

BACKGROUND: In preterm infants, the amplitude-integrated electroencephalogram (aEEG) is not established in clinical routine. The aim of this study was to derive normative data on aEEG parameters by means of longitudinal characterization and to evaluate the impact of gestational age (GA), postnatal age (PNA), postmenstrual age, sedation, and patent ductus arteriosus (PDA).

METHODS: Recordings from 61 infants with GA 28-31 weeks were obtained during the first $72 \mathrm{~h}$, then weekly until the age of 4 wk. Infants were divided into three groups: (i) no sedation, no PDA, (ii) sedation, no PDA, and (iii) sedation, PDA. Assessed parameters included background activity, cycling, amplitude, and log ratio of the maximum/minimum amplitude.

RESULTS: GA and PNA had a significant impact within $72 \mathrm{~h}$. Sedation modified aEEG, and presence of PDA was associated with reduced aEEG scores within $72 \mathrm{~h}$. The log ratio of the amplitude correlated with GA but was unaffected by sedation and PDA.

CONCLUSION: Evaluation of electrocortical background activity within the first postnatal hours and longitudinally over days and weeks is important to better understand the postnatal factors impacting cerebral function in preterm infants. There is a need to agree on definitions and a standardized reporting system in order to permit comparisons between studies and establish aEEG as a method for routine monitoring of preterm infants.

A cquired brain injury constitutes a significant threat to the developing preterm infant. Biomarkers measured at or soon after birth may be useful in diagnosing and predicting complications and future outcome. Several studies have evaluated whether, and to what extent, amplitude-integrated electroencephalogram (aEEG)-derived parameters can be used for the identification of neurological dysfunction and prediction of outcome in preterm infants (1-7). The increasing adoption of the aEEG method in the neonatal intensive care unit is reflected by the growing number of studies published during the past decade, although these still do not match the knowledge that was gained in term-born infants over the same period of time (8-10). Efforts have been made to define normative values and changes in aEEG parameters with respect to gestational age (GA) and postmenstrual age (PMA) (11-15), and recently, integrative studies correlating aEEG with various clinical parameters have been published (16-18). These examples demonstrate that the concept of aEEG monitoring can be very useful in preterm infants. However, there are several challenges that delay the translation of aEEG monitoring into clinical practice in preterm infants. A major obstacle when comparing results from different studies is due to the differences in study populations, evaluated time periods, aEEG parameters, outcome assessments, and statistical reporting $(7,19-21)$. A second concern is the influence on the electrocortical activity from extracerebral factors and from parameters inherent to the care and the physiology of the preterm infant $(18,22-25)$. The exploration of early electrocortical activity, as recorded within the first hours and days, serves as the closest estimate of intrauterine electrocortical function and also contributes to a better understanding of developmental brain injury. However, whereas a series of studies evaluated the development of aEEG patterns in the initial days after birth $(11,20,21)$, continuous monitoring of changes in electrocortical activity during the first postnatal hours has received much less attention $(1,2,13)$. Besides these essential clinical considerations, the appropriate definition of the measurement time point has implications, particularly for studies hypothesizing on the prognostic abilities of aEEG monitoring. Together with the initial observations about the duality of intrauterine (GA) and extrauterine (postnatal age (PNA)) maturation and recent reports on repeated aEEG measurement after birth, aEEG profiles are expected to involve complex and competitive changes in this critical period. In the future, we should be able to better assess the neurological condition and risk for infants as soon as they are admitted to the neonatal intensive care unit by adding aEEG technology to our panel of monitoring instruments.

The aim of the current study was to:

1. Achieve a comprehensive longitudinal characterization of aEEG development during the first $4 \mathrm{wk}$ of life in preterm infants born at a GA of 28-31 wk and without evidence of neonatal brain injury;

2. Compare the effects of GA, PNA, and PMA on aEEG parameters; 
3. Compare and validate commonly used aEEG parameters and scores;

4. Establish statistical data analyses for the evaluation of longitudinally aEEG-derived data; and

5. Analyze the impact of sedation and patent ductus arteriosus (PDA) on aEEG parameters.

\section{RESULTS}

\section{Visual Background}

The odds in favor of observing continuous activity increased with GA (odds ratio $(\mathrm{OR})=2.37(1.43-3.92) ; P<0.01)$ and PNA $(\mathrm{OR}=2.19(1.81-2.65) ; P<0.01)$. The proportion of infants with continuous activity increased from $9 \%$ to $22 \%$ from $6-12 \mathrm{~h}$ to $66-72 \mathrm{~h}$ and increased further at week $1(75 \%)$ and at week 4 (100\%). Only PMA was associated with the presence of continuity $(\mathrm{CO})$ at $1-4 \mathrm{wk}$; the odds for observing $\mathrm{CO}$ increased by 2.80 $(1.55-5.04)(P<0.00001)$ for each additional week. Burst suppression pattern was present only during the first $72 \mathrm{~h}$ and was exclusively associated with analgosedation $(P<0.01)$ (Figure 1 ).

\section{Cycling: First Sinusoidal Variation}

The time from birth to the appearance of first sinusoidal variation (FSV) is graphed in Figure 2a. The time to FSV revealed a significant association with GA $(\mathrm{LRT}=3.91 ; d f=1 ; P<0.05)$ and study group ( $\mathrm{LRT}=7.79 ; d f=2 ; P<0.01)$. For each additional week of gestation, time to FSV was reduced by a factor of 1.25 (1.00-1.59). After adjusting for GA, the average predicted time to FSV was $1.93(1.16-3.20)$ times higher in the sedation group $($ mean $=13.8 \mathrm{~h} ; n=17$; adjusted mean at $30 \mathrm{wk}$

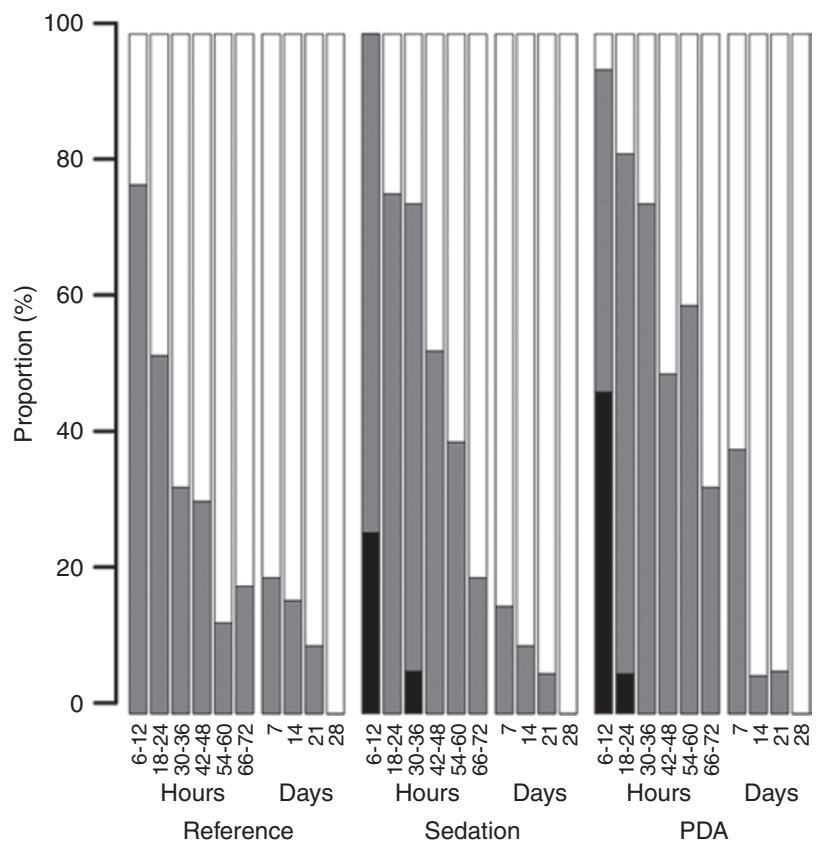

Figure 1. Visual background annotation for each monitoring period and study group. Black bars, proportion of burst suppression; gray bars, discontinuous; white bars, continuous. The number of patients is 21 in the reference group, 20 in the sedation group, and 20 in the PDA group. All forms of burst suppression are pooled into one group. PDA, patent ductus arteriosus. of gestation $=14.1 \mathrm{~h})$ than that in the reference group $($ mean $=$ $6.4 \mathrm{~h} ; n=17$; adjusted mean at $30 \mathrm{wk}=7.3 \mathrm{~h}$ ). Time to FSV in the PDA group (mean $=16.6 \mathrm{~h} ; n=20$ ) was found to be similar to that in the sedation group.

\section{Cycling: First Obvious Widening}

The proportional hazard model revealed a positive association between the first obvious widening period and GA (LRT
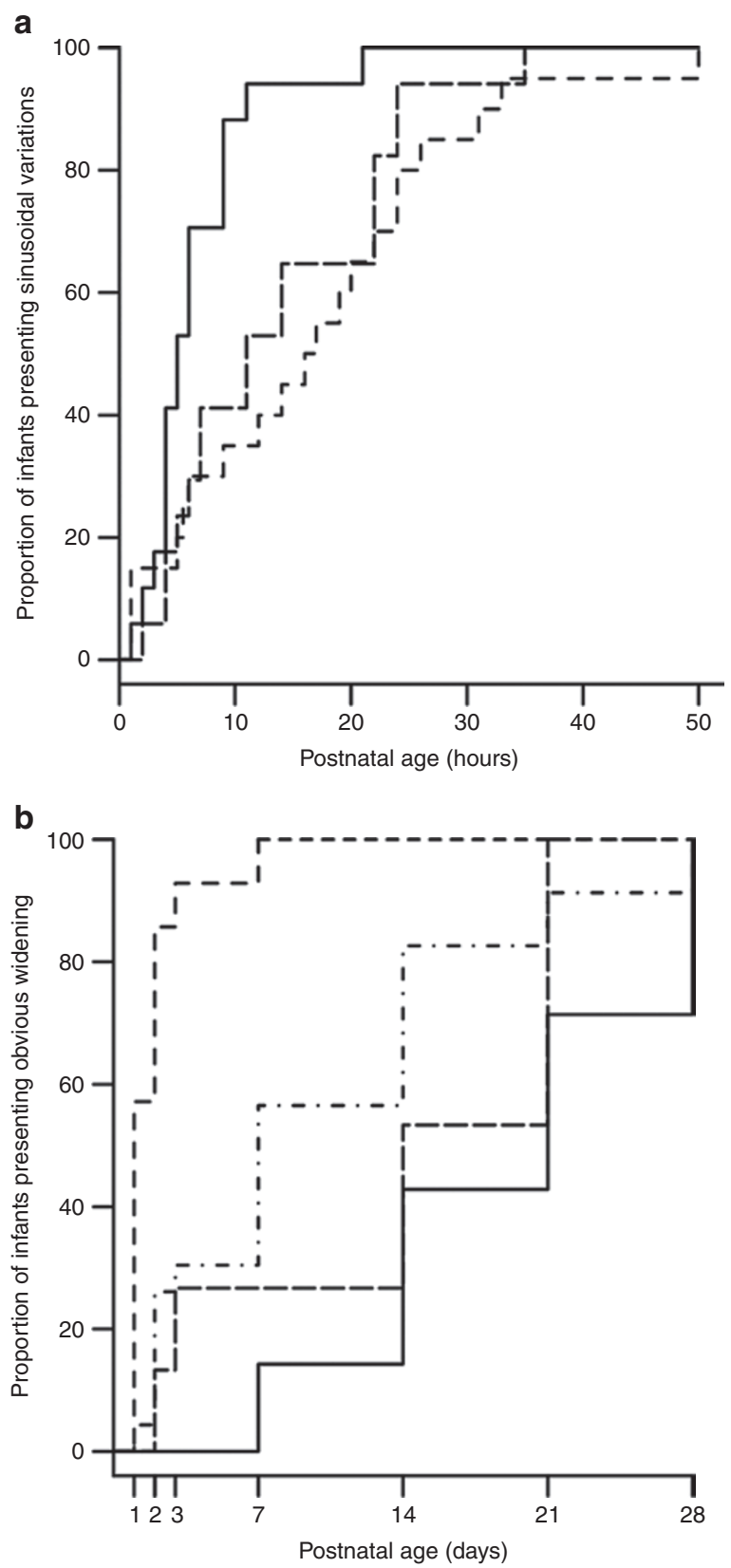

Figure 2. Cycling. (a) Cumulative proportions of infants for whom tracing shows sinusoidal variations across the three study groups. The number of patients is 17 in the reference group (solid line), 17 in the sedation group (dashed line), and 20 in the PDA group (short-dashed line). (b) Cumulative proportions of infants for whom tracing shows obvious widening across GA groups (GA: $28 \mathrm{wk}$, solid line, $n=7 ; 29 \mathrm{wk}$, dashed line, $n=15 ; 30 \mathrm{wk}$, dashed-dotted line, $n=23 ; 31 \mathrm{wk}$, short-dashed line, $n=14$ ). GA, gestational age; PDA, patent ductus arteriosus. 
a

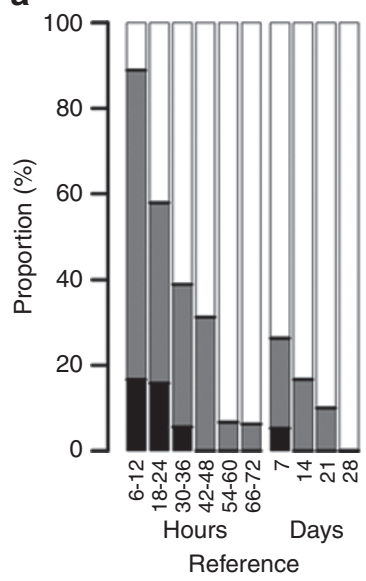

C

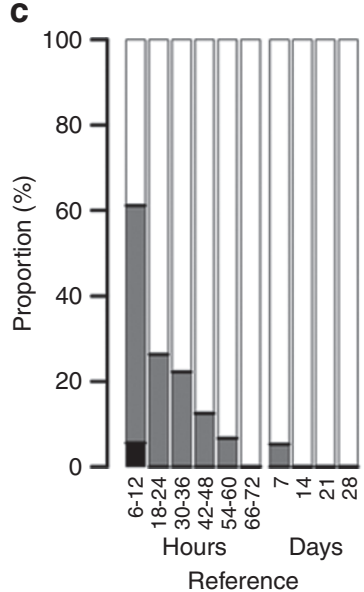

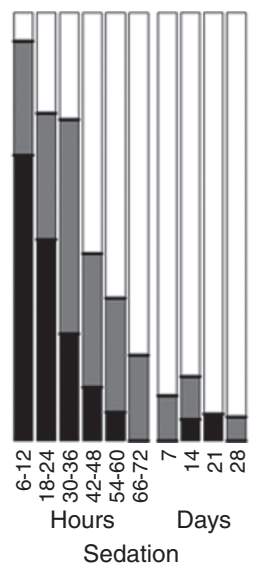
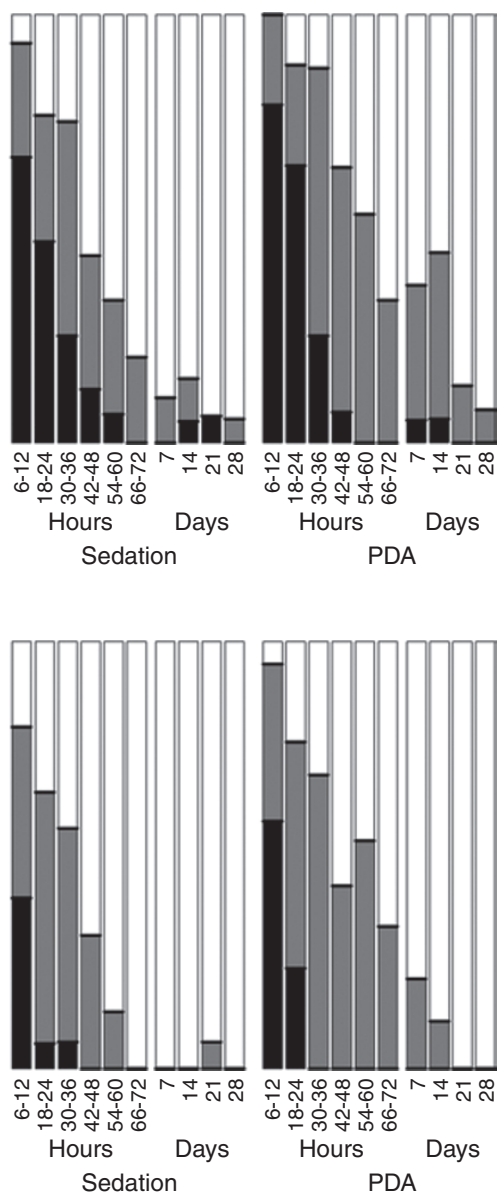

b
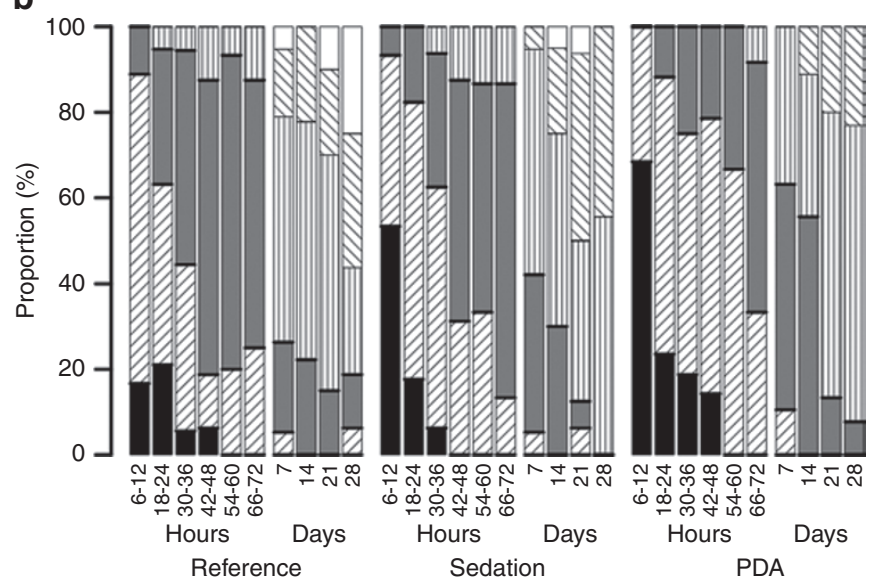

d
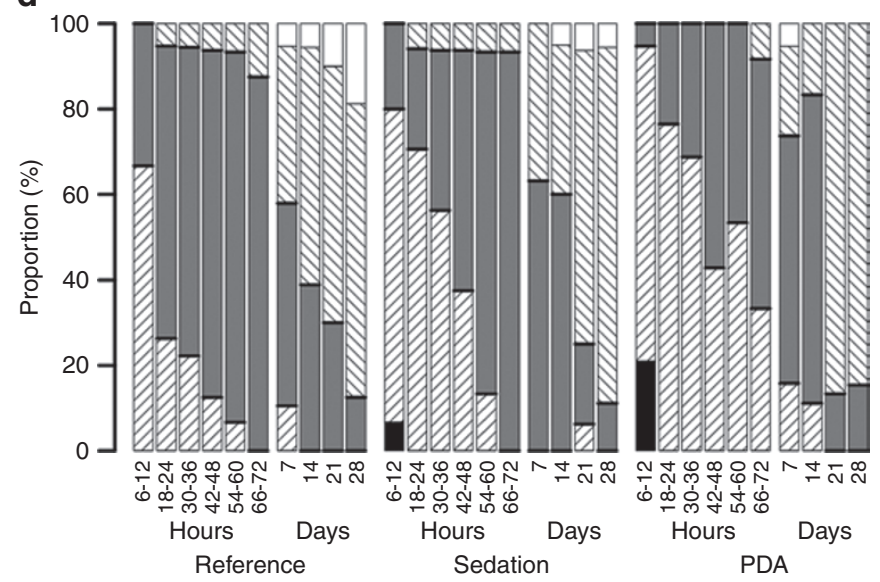

Figure 3. Distributions of individual Burdjalov scores for each monitoring period and study group. (a) CO, (b) CY, (c) ALB, and (d) BS-ALB. Each figure panel is divided into three parts according to the three treatment groups (reference, $n=21$; sedation, $n=20 ;$ and PDA, $n=20$ ). The different time points of aEEG evaluation are shown for each treatment group as marked on the x-axis. The sum score for each parameter is colored as follows: (a) CO: black bar $=0$, gray bar $=1$, and white bar $=2 ;(\mathbf{b}) C Y$ : black bar $=0$, right diagonal patterned bar $=1$, gray bar $=2$, vertical patterned bar $=3$, left diagonal patterned bar = 4, and white bar = 5; (c) ALB: black bar =0, gray bar = 1, and white bar = 2; and (d) BS+ALB: black bar = 0, right diagonal patterned bar = 1, gray bar $=2$, left diagonal patterned bar =3, and white bar = 4. aEEG, amplitude-integrated electroencephalogram; ALB, amplitude of the lower border; BS-ALB, bandwidth span and amplitude of the lower border; CO, continuity; CY, cycling; PDA, patent ductus arteriosus.

$=24.7 ; d f=1 ; P<0.01)$. A first obvious widening was present within the first $3 \mathrm{~d}$ in $90 \%$ of the infants with a GA more than $31 \mathrm{wk}$ (Figure $2 \mathrm{~b}$ ), whereas this proportion was only reached at weeks 3-4 in less-mature infants.

\section{Parameters of Burdjalov Score}

Distributions of the individual parameters in the three groups are represented in Figure 3a-d.

\section{Continuity}

During the first $72 \mathrm{~h}, 43 \%$ of traces were labeled as continuous $(\mathrm{CO}=2), 35 \%$ were assessed as somewhat continuous $(\mathrm{CO}=1)$, and $22 \%$ as discontinuous ( $\mathrm{CO}=0)$. In total, $87 \%$ of discontinuous patterns were observed within $36 \mathrm{~h}$ (Figure 3a). During this period, the odds in favor of observing a somewhat continuous or continuous pattern (CO scores 1 or 2) increased with PNA $(\mathrm{OR}=2.71(1.81-4.06) ; P<0.01)$ but not with $\mathrm{GA}(\mathrm{OR}=1.57$ (0.91-2.69); $P>0.05)$. The odds ratio for observing $\mathrm{CO}$ (scores 1 or 2) was $6.48(1.28-32.9)$ times lower in sedated infants than in the reference group. Between 2 and $4 \mathrm{wk}$, presence of $\mathrm{CO}$ was associated with GA $(\mathrm{OR}=2.62(1.36-5.03) ; P<0.01)$ and PNA $(\mathrm{OR}=1.66(0.99-2.76) ; P<0.05)$.

\section{Cycling}

Immature cycling $(\mathrm{CY} \leq 2)$ appeared in 95\% of infants during the first $72 \mathrm{~h}$, and only $5 \%$ had regular and mature patterns. Total absence of cycling $(\mathrm{CY}=0)$ was associated with analgosedation (Figure $3 \mathbf{b})$. Of note, emergence of cycling $(\mathrm{CY} \geq 2$ ) over the first $72 \mathrm{~h}$ was not affected by analgosedation. By contrast, manifestation of cycling patterns depended on PNA $(\mathrm{OR}=$ 2.48 (1.92-3.21); $P<0.01)$ and GA $(\mathrm{OR}=2.35(1.37-4.04) ; P<$ 0.05). After week 1, 90\% of infants showed cycling (CY > 3). Using proportional odds logistic regression including all cycling scores, two main predictors for the development of cycling were identified: $\mathrm{GA}(\mathrm{OR}=2.41(1.80-3.23) ; P<0.01)$ and $\mathrm{PNA}(\mathrm{OR}=$ 2.21 (1.70-2.88); $P<0.01)$. Each additional postnatal week was associated with a $2.37(1.89-2.96)$-fold $(P<0.01)$ increase in the likelihood of reporting a higher cycling score. 


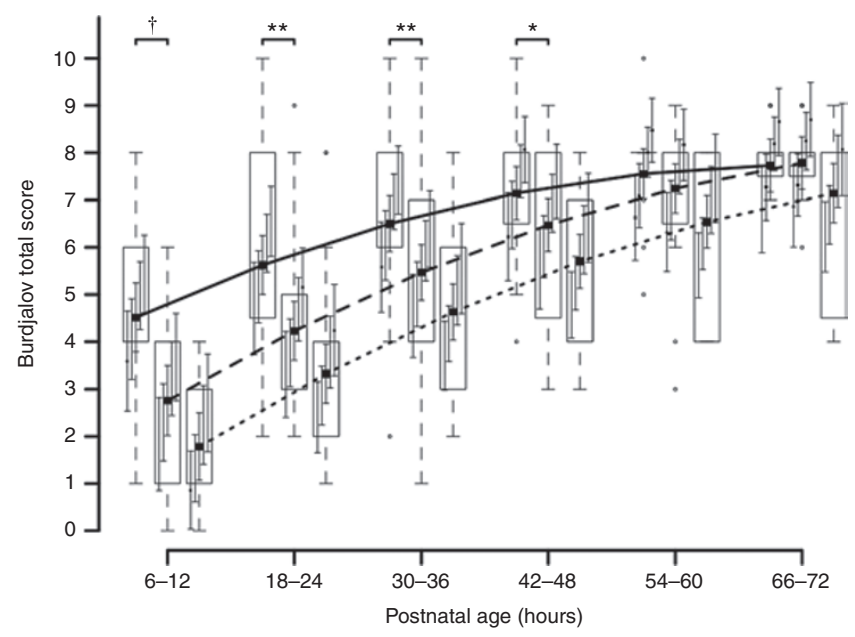

Figure 4. Total Burdjalov score over the first monitoring period. Actual distributions are shown as shaded gray boxplots. Vertical lines correspond to the predicted total score and $95 \%$ confidence intervals for $\mathrm{GA}=28,29$, 30 , and 31 for each study group at each measurement time. The number of patients is 18 in the reference group (solid line), 17 in the sedation group (dashed line), and 19 in the PDA group (dotted line). Significance levels: ${ }^{\dagger} P<0.0001,{ }^{* *} P<0.01,{ }^{*} P<0.05$. GA, gestational age; PDA, patent ductus arteriosus.

\section{Amplitude of the Lower Border}

Severely depressed traces (amplitude of the lower border (ALB) $=0)$ were detected only within the first $24 \mathrm{~h}$ (35\% of traces at $6-12 \mathrm{~h}$ and $9 \%$ at $18-24 \mathrm{~h}$; Figure $3 \mathrm{c}$ ), and the main contributor was PNA $(\mathrm{OR}=2.25(1.79-2.82) ; P<0.01)$. During the 6-12-h period, the likelihood of presenting a score of 0 , rather than 1 or 2 , depended on the study group (LRT $=8.02$; $d f=2$; $P<0.05)$; sedation increased the odds for detecting severely depressed traces by a factor of $7.98(1.40-85)(P<0.05)$; and PDA was not found to be significant $(\mathrm{OR}=1.98(0.5-7.8)$; $P>0.05)$. The proportion of infants with elevated lower border $(A L B=2)$ increased from $21 \%$ at $6-12 \mathrm{~h}$ to $91 \%$ at $66-72 \mathrm{~h}$, and the main contributor was GA $(\mathrm{OR}=1.81(1.04-3.15) ; P<0.04)$. Four infants showed a somewhat depressed ALB score at week 1 that could only be associated with PDA status (Barnard's test, $P<0.05$ ) regardless of $\mathrm{GA}$.

\section{Bandwidth Span and Amplitude of the Lower Border}

In the first monitoring period (6-72h), scores of $1(41.5 \%)$ and $2(52.6 \%)$ were characteristic of this combined parameter (Figure 3d). The occurrence of a score $>1$ increased by a factor of $2.25(1.71-2.97)(P<0.01)$ for every $12-\mathrm{h}$ increase in PNA, a

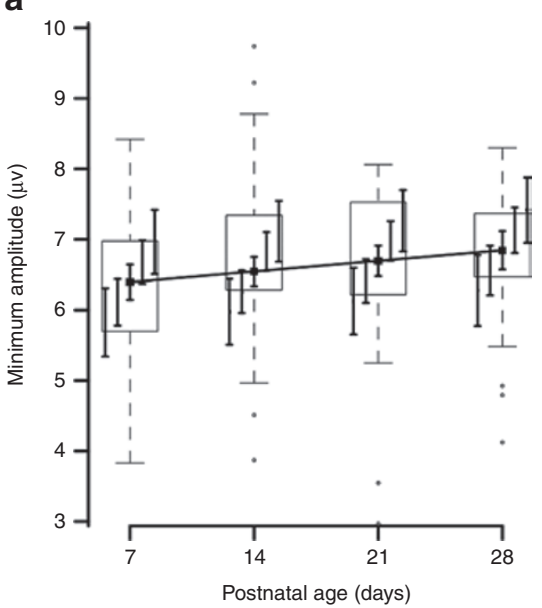

b

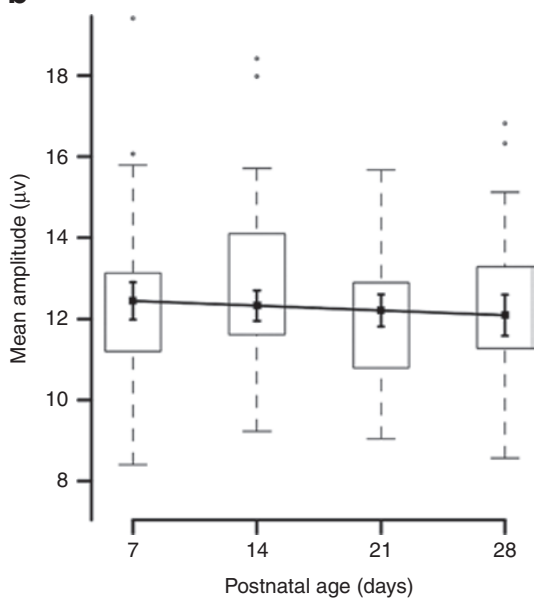

C

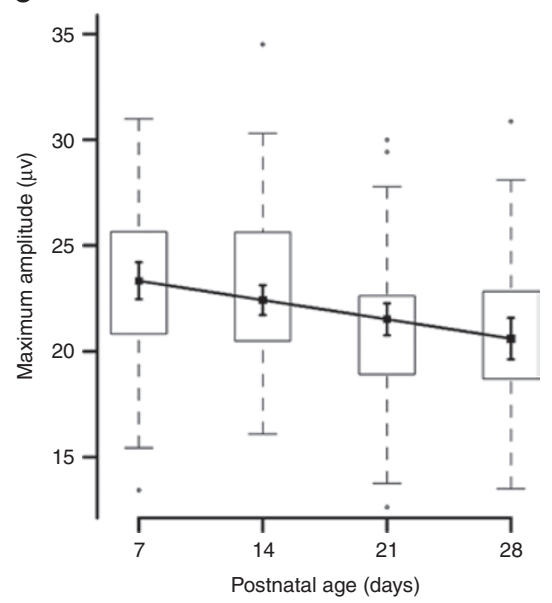

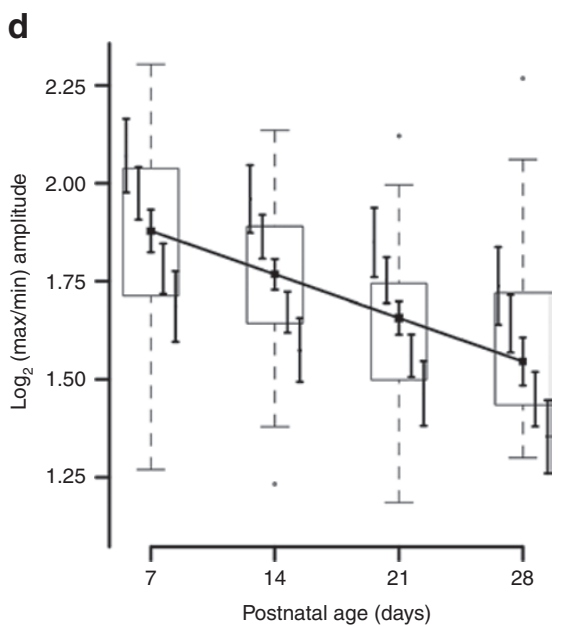

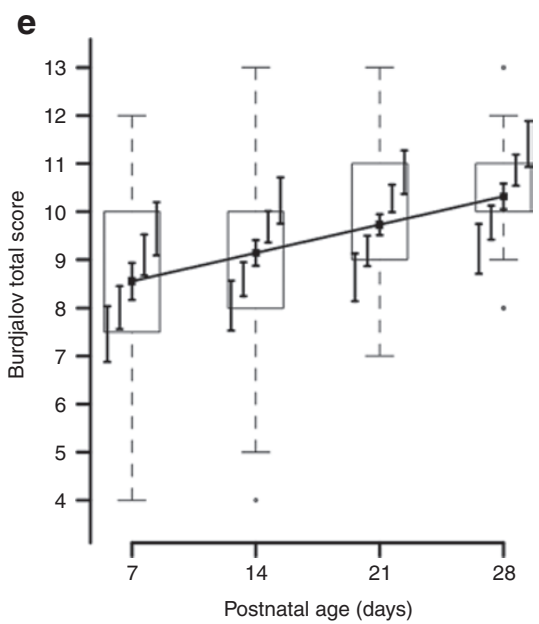

Figure 5. Amplitude parameters over the second monitoring period. (a-d) Distribution of amplitude parameters during the 7- to 28-day monitoring period. (e) The Burdjalov total score during the 7- to 28-day monitoring period. Actual distributions are shown as shaded gray boxplots. Vertical lines correspond to the predicted total score and $95 \%$ confidence intervals for $\mathrm{GA}=28,29,30$, and 31 . 
a

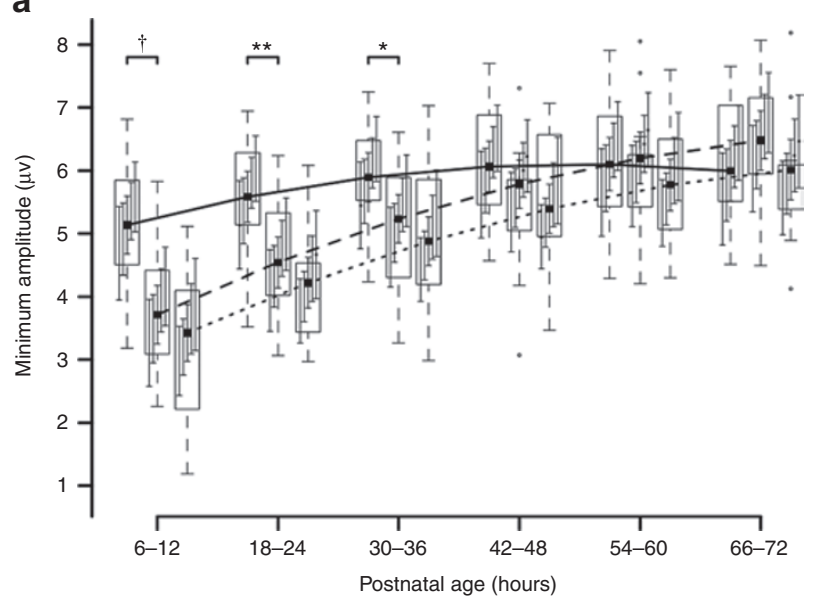

C

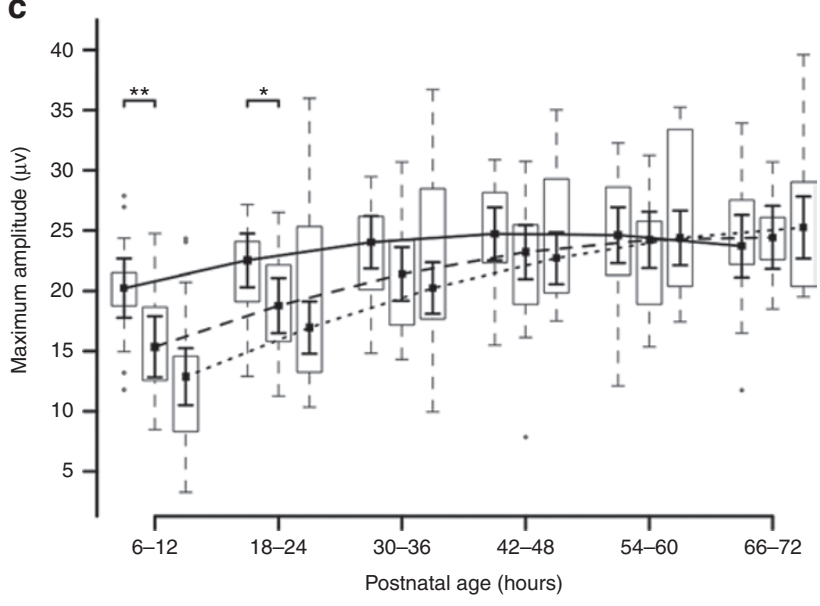

b

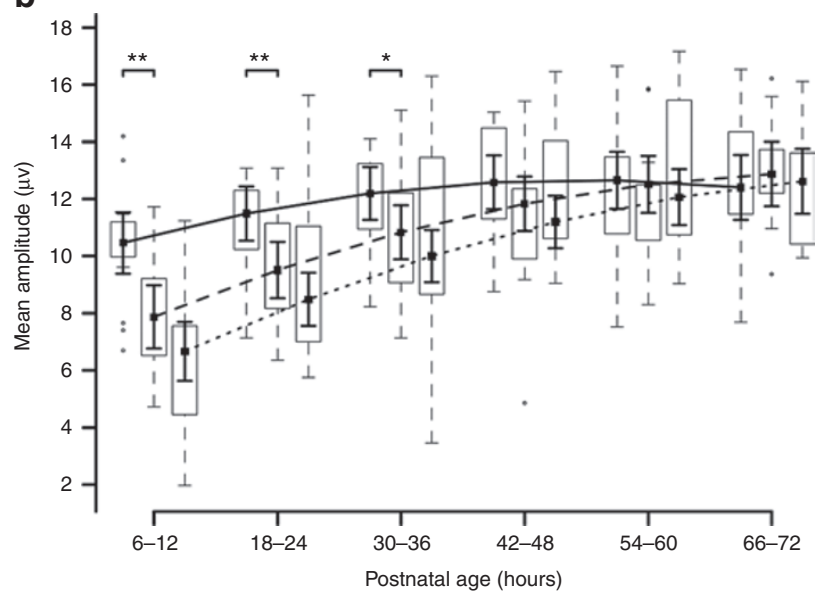

d

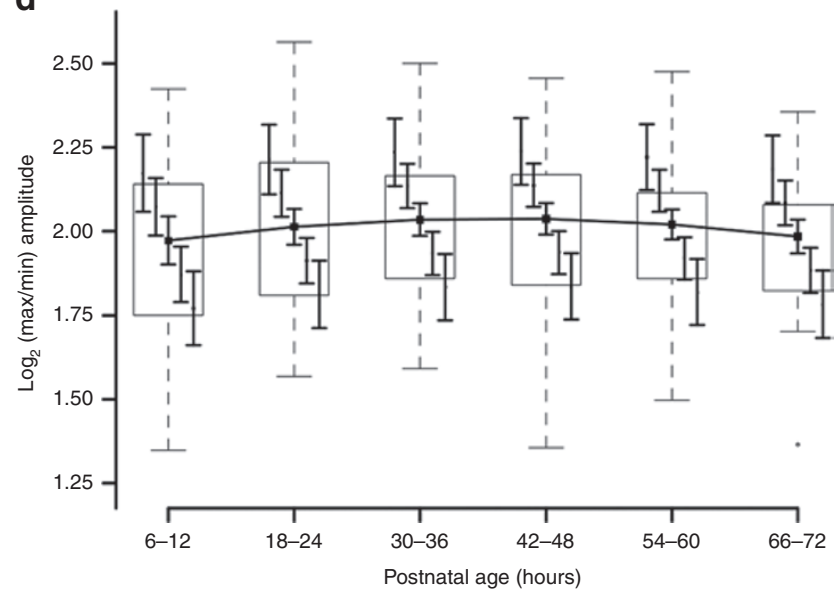

Figure 6. Amplitude parameters over the first monitoring period: (a) minimum amplitude, (b) mean amplitude, (c) maximum amplitude, and (d) maximum/minimum ratio. Actual distributions at each measurement time are shown as shaded gray boxplots. Given model term significance, fitted models are shown as dark lines. The number of patients is 18 in the reference group (solid line), 17 in the sedation group (dashed line), and 19 in the PDA group (dotted line). Significance levels: ${ }^{\dagger} P<0.0001,{ }^{* *} P<0.01,{ }^{*} P<0.05$.

and by a factor of $1.93(1.18-3.14)(P<0.01)$ for each additional week of gestation. The presence of a score $>1$ was reduced by a factor of $3.08(0.98-9.65)(P<0.05)$ in the sedation group, whereas the PDA group did not have an increased risk for immature traces $(\mathrm{OR}=0.72(0.22-2.39) ; P>0.05)$. During the first $3 \mathrm{~d}$, bandwidth span and amplitude of the lower border was largely dominated by the emergence of maturing (52.6\%) and mature traces (5.2\%) from immature traces (38.4\%).

\section{Total Score}

Distribution of the Burdjalov total score (TS) over the first $72 \mathrm{~h}$ is displayed in Figure 4. The TS increased significantly with GA (LRT $=8.96 ; P<0.01)$ and PNA $(P<0.01)$. As compared with the reference group, the TS was significantly lower in the sedation group during the first $48 \mathrm{~h}$ and significantly lower in the PDA group during the first $72 \mathrm{~h}(P<0.05)$. After week 1 (Figure 5e), the TS increased significantly with PMA, regardless of group allocation. Our data show a slope of 0.58 (0.45-0.72), which is slightly lower than that in the original paper by Burdjalov et al. (estimated slope to be $\sim 0.70-0.75$ over 23-37 GA wk).

\section{Amplitude Parameters}

The minimum, mean, and maximum amplitudes for the first $72 \mathrm{~h}$ are presented in Figure 6a-d, and for weeks 1-4 in Figure 5a-d. Minimum amplitude was the only parameter affected by GA (LRT $=3.77 ; d f=1 ; P<0.05)$, increasing by $0.22(-0.01$ to 0.45$) \mu \mathrm{V}$ per additional week of GA. All amplitude parameters are characterized by a nonlinear relationship with PNA consisting of a sharp increase in the first postnatal hours and behaviors specific to the different study groups. Impact of the latter decreased in the order of minimum $(\mathrm{LRT}=9.6 ; P<0.01)$, mean $(\mathrm{LRT}=9.4 ; P<0.01)$, and maximum (LRT $=5.2 ; P=0.07)$ amplitudes. For all three parameters, the effect of the study groups was found to be time dependent (all $P<0.01$ ). In particular, infants from the sedation group presented lower minimum and mean amplitudes up to $36 \mathrm{~h}$ $(P<0.05)$. The PDA group had a predicted minimum amplitude that was $0.23(-0.14$ to 0.90$) \mu \mathrm{V}$ lower than the reference group. During weeks $1-4$, the minimum amplitude increased at a rate of $0.19(0.09-0.29) \mu \mathrm{V}$ per additional week, whereas the maximum amplitude decreased at a rate of $-0.75(-1.12$ to -0.38$) \mu \mathrm{V}$ for each additional postnatal week (Table 1). The mean amplitude remained constant at $\sim 12.3(11.9-12.7) \mu \mathrm{V}$. The log ratio between 
Table 1. Regression coefficients used to model Burdjalov total score and amplitude parameters over the 7-to 28-day period

\begin{tabular}{lccccc}
\hline Parameter & Total score & Minimum & Mean & Maximum & $\begin{array}{c}\text { Maximum } / \text { minimum } \\
\text { amplitude ratio }\end{array}$ \\
\hline Gestational age & $0.54(0.33-0.75)^{* *}$ & $0.28(0.08-0.48)^{* *}$ & NS & NS & $-0.09(-0.13 \text { to }-0.05)^{* *}$ \\
Postnatal age & $0.58(0.43-0.74)^{* *}$ & $0.14(0.04-0.25)^{* *}$ & NS & $-0.9(-1.3 \text { to }-0.5)^{* *}$ & $-0.11(-0.13 \text { to }-0.08)^{* *}$ \\
Postmenstrual age & $0.58(0.45-0.71)^{* *}$ & $0.19(0.09-0.29)^{* *}$ & $-0.03(-0.21$ to 0.15$)$ & $-0.75(-1.11 \text { to }-0.38)^{* *}$ & $-0.1(-0.13 \text { to }-0.08)^{* *}$ \\
\hline
\end{tabular}

One time unit corresponds to 1 wk. Model including PMA is given only in addition to the model that can include GA and PNA. $95 \%$ Confidence intervals in brackets.

Significance levels: ${ }^{* *} P<0.01$.

GA, gestational age; PMA, postmenstrual age; PNA, postnatal age; NS, not significant.

Table 2. Patient characteristics

\begin{tabular}{|c|c|c|c|c|c|}
\hline Parameter & $\begin{array}{c}\text { Reference group } \\
\text { (no sedation and no PDA }(n=21) \text { ) }\end{array}$ & $\begin{array}{l}\text { Sedation group (sedation } \\
\text { and no PDA }(n=20))\end{array}$ & $\begin{array}{c}P \text { value } \\
\text { (sedation) }^{\mathrm{a}}\end{array}$ & $\begin{array}{l}\text { PDA group (sedation } \\
\text { and PDA }(n=20))\end{array}$ & $\begin{array}{l}P \text { value } \\
(\text { PDA })^{b}\end{array}$ \\
\hline Gestational age (wk) & $30.7(0.952)(28.6-31.8)$ & $30.1(0.904)(28.1-31.8)$ & $0.052(0.061)$ & $29.5(0.8)$ & $0.029(0.021)$ \\
\hline Umbilical cord pH & $7.31(0.05)(7.25-7.42)$ & $7.32(0.05)(7.22-7.46)$ & $0.709(0.762)$ & $7.27(0.10)(7.01-7.42)$ & $0.108(0.282)$ \\
\hline Apgar at $1 \mathrm{~min}$ & $7(5-9)$ & $6(4-8)$ & $<0.001(0.001)$ & $6(2-8)$ & $0.401(0.537)$ \\
\hline $\begin{array}{l}\text { Continuous positive } \\
\text { airway pressure } \\
\text { treatment ( } \mathrm{h} \text { ) }\end{array}$ & $5.11(8.03)(0-28)$ & $7.1(6.12)(1-26)$ & $0.379(0.014)$ & 7.96 (4.96) (0.8-19) & $0.626(0.386)$ \\
\hline Ventilator treatment $(\mathrm{d})$ & $0.3(1.3)(0-6)$ & $13.3(27.2)(0-122)$ & $0.034(<0.001)$ & 19.9 (31.6) (0-138) & $0.483(0.156)$ \\
\hline Male $(n)$ & 12 & 14 & 0.273 & 11 & 0.263 \\
\hline Sepsis (n) & 0 & 3 & 0.036 & 0 & 0.043 \\
\hline Ketamine $(n)$ & - & 12 & - & 8 & 0.27 \\
\hline Fentanyl derivatives $(n)$ & - & 15 & - & 12 & 0.348 \\
\hline Benzodiazepine ( $n)$ & - & 10 & - & 12 & 0.242 \\
\hline Opioid antagonist $(n)$ & - & 2 & - & 5 & 0.102 \\
\hline
\end{tabular}

Mean, median (Apgar), SD and range (in brackets), and number of patients for each possible group status are shown. $P$ values are calculated by means of $t$-test and Mann-Whitney test (in brackets) for the continuous parameters and according to Barnard for the categorical parameters.

aResults of comparing the sedation and reference groups. ${ }^{b}$ Results of comparing the PDA and sedation groups.

the maximum and minimum amplitudes was computed to obtain an estimate of the dynamic range of variation of the aEEG trace. This $\log$ ratio was significantly correlated with GA in the first $72 \mathrm{~h}$ and was not impacted by PDA or sedation. In the second monitoring phase, the log ratio decreased with PMA at a rate of -0.11 $(-0.13$ to -0.09$)$ per week.

\section{Medication}

Although medication regimens were reasonably balanced across the study groups (Table 2), we examined whether the residual variance patterns could be associated with specific sedative drugs. For illustration, we report the aEEG maximum amplitude in infants who were administered a combination of fentanyl with benzodiazepine $(n=9)$ or ketamine $(n=9)$. This parameter was dramatically attenuated in the benzodiazepine group for up to $60 \mathrm{~h}$ after administration (Figure 7). A similar trend was observed for the mean amplitude, although found significant only at 30-36h
$(P<0.05)$, but not for any other parameters including minimum amplitude or Burdjalov individual and TSs.

\section{DISCUSSION}

This study provides a comprehensive analysis and comparison of postnatal longitudinally obtained aEEG parameters from the first postnatal hours until the age of $4 \mathrm{wk}$ in a cohort of preterm infants born at 28-31 gestational weeks. Our results confirm previous studies by demonstrating the emergence of continuous patterns, cycling, and ALB on the aEEG signal with increasing age $(14,15,20)$. We expand this knowledge by (i) including the first 72 postnatal hours in the analysis, (ii) comparing the established and novel aEEG parameters in a single study, and (iii) focusing on the age group of 28-31 wk GA, in which these data were lacking. We obtained reference data, compared the different parameters extracted from the aEEG, and evaluated potential confounders. This has major clinical relevance 


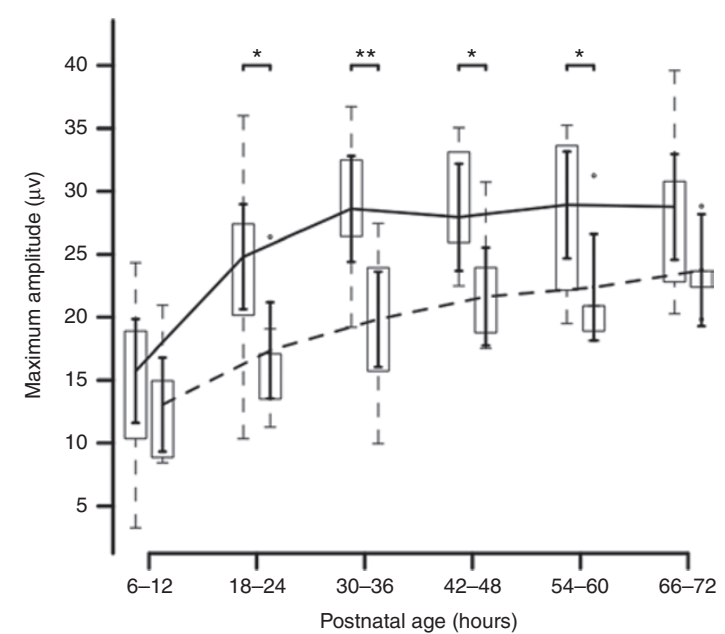

Figure 7. Impact of medication on maximum amplitude during the first $72 \mathrm{~h}$ postnatal. Actual distributions are shown as shaded gray boxplots. Vertical lines correspond to the predicted maximum amplitude of $95 \%$ confidence intervals for infants who received ketamine (solid line, $n=8$ ) or benzodiazepine (dashed line, $n=9$ ). Significance levels: ${ }^{*} P<0.01,{ }^{*} P<0.05$.

because alterations in these parameters are speculated to be potential biomarkers of good or adverse neurological outcome (5) that are already well established in term newborns (10). In the current study, GA and PNA had a distinct impact on aEEG parameters. Maturation of cycling was seen mainly during the first weeks, but less during the first $72 \mathrm{~h}$ of life. An additional parameter, which has previously been discussed as the strongest developmental indicator, is the minimum amplitude (1). The minimum amplitude increased within the first $72 \mathrm{~h}$ after birth and increased further during the first weeks of life, whereas the maximum amplitude decreased, expressing the compression of bandwidth span with increasing GA. The GA is an important factor, because almost always it is presented as a discrete measure, usually in $2-4$-wk periods $(6,14,20)$. To the best of our knowledge, the current study is the first in which the exact GA in weeks was modeled against the evaluated aEEG parameter. With increasing PNA/PMA, the mean amplitude remained constant, whereas the minimum amplitude and the maximum amplitude were affected. Although amplitude is a valid and objective parameter, it must be interpreted with caution because of its susceptibility to artifacts, environmental and iatrogenic factors $(20,24,26)$. We therefore introduced the log ratio between the maximum and minimum amplitudes to obtain an estimate of the dynamic range of variation of the aEEG trace. Because this log ratio was significantly correlated with GA in the first $72 \mathrm{~h}$ and was not impacted by PDA or sedation, this parameter permits GA to be estimated independently of potential confounders such as sedation. Sedation resulted in an overall depressed aEEG background and lower scores during the first $24-36 \mathrm{~h}$. The effect on aEEG parameters of this sedation, given as a bolus in conjunction with intubation after delivery, was time dependent and decreased over time. Of note is the already mentioned lack of impact of sedation on the log ratio of maximum and minimum amplitude. Within the sedation group, we were able to demonstrate that suppression of
aEEG parameters is more pronounced in benzodiazepine- than in ketamine-treated newborns. This underlines the clinical importance of a detailed knowledge of the effects of administered drugs to avoid misinterpretation of aEEG parameters.

In addition to sedation, we evaluated the impact of PDA on electrocortical activity. Infants with PDA showed reduced Burdjalov TSs in the first $72 \mathrm{~h}$ postnatal as compared with the sedation group. The difference between the sedation group and the PDA group persisted at postnatal week 1, a time when the duct was closed, suggesting either long-term impact of PDA or an ibuprofen effect on electrocortical activity. This is in contrast to several studies that did not find notable changes in (a)EEG patterns before or after closure of the ductus arteriosus despite variations in cerebral blood flow velocity, volume, or oxygenation described in infants with PDA $(27,28)$. Of note, our study was not designed to evaluate potential mechanisms. Therefore, we can only speculate on the reasons for these different observations.

The current study has limitations. aEEG recordings were not commenced immediately after birth, which would be the closest time point to extrauterine transition. However, stabilization, neonatal care, and minimal handling were more important. Nevertheless, the majority of recordings, including all infants from a geographically determined region (highly representative), were started within $6 \mathrm{~h}$ after birth. Another limitation of this study is that to date, long-term outcome has not been analyzed. One of the main findings of our study is that aEEG is influenced by sedation during the first days of life, but further studies are necessary to investigate the influence of sedation on long-term outcome in these children.

\section{Conclusion}

The aEEG has been increasingly used in neonatology in recent years. Several studies evaluated the potential of the aEEG as a biomarker of brain injury and predictor of neurodevelopmental outcome. The advantages of aEEG as compared with bloodbased biomarkers are multiple. However, if aEEG is ultimately to be established for these purposes, there is an urgent need to agree on common definitions, relevant parameters, and a standardized reporting strategy. Because extrauterine life is a pathological condition for preterm infants, defining normality of electrocortical activity is a challenge, especially because of the great variance in preterm conditions. Longitudinal evaluation of infants receiving clinical treatment and presenting epidemiology is needed to avoid persistent variations in aEEG parameters. Our methodology and results could form the basis for the development and establishment of a standardized reporting system, providing raw data for statistical analysis and the exchange of aEEG data between centers.

\section{METHODS}

This study was performed at the neonatal intensive care unit of the Department of Pediatrics II, University Hospital of Innsbruck, Austria. Inborn infants with a GA between 28 and 31 completed wk, delivered between October 2007 and August 2010, were eligible for the study. This age group of early preterm infants was chosen because they form the majority of preterm infants admitted to our unit, thus allowing a sufficient number of infants to be recruited during a limited time period and thereby avoiding potential confounders such as changes 
Table 3. Synopsis of aEEG parameters considered in this study

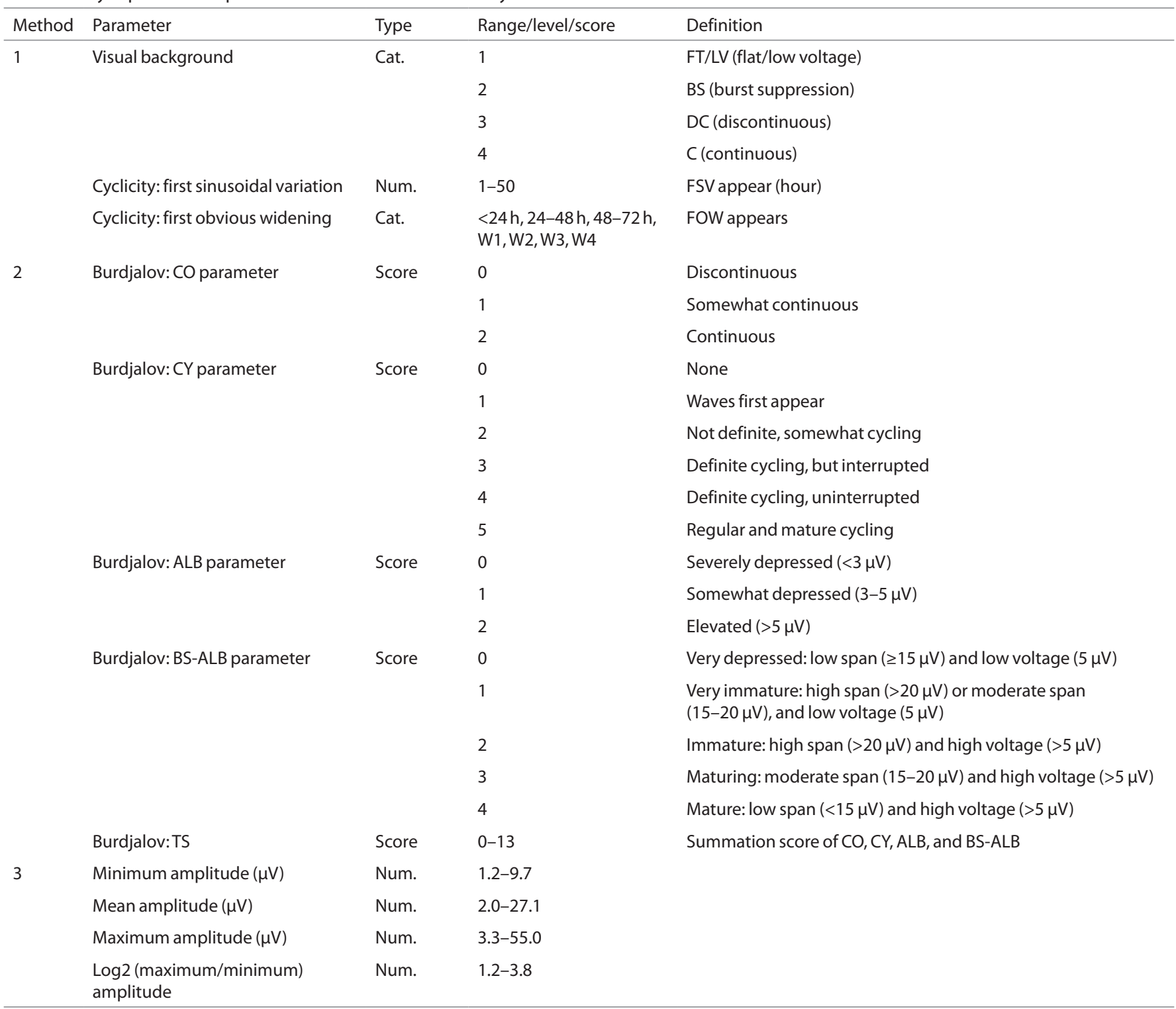

Type: data type whether the parameter is numerical (Num.), categorical (Cat.), or score. Range: possible values for the parameter (categorical or score) or range of variation (numerical). ALB, amplitude of the lower border; BS-ALB, bandwidth span and amplitude of the lower border; CO, continuity; CY, cycling; FOW, first obvious widening; FSV, first sinusoidal variation; TS, total score; VB, visual background; W, week.

in neonatal care and treatment. GA was assessed by obstetrical diagnostic estimate and postnatal clinical examination using the modified Ballard Score. PMA and PNA were recorded for all infants. Preterm infants were recruited after obtaining written consent from at least one parent, either prenatally or within the first $6 \mathrm{~h}$ after birth. Exclusion criteria were congenital malformations, congenital infections, arterial hypotension (on the basis of published normative data) (29), need for continuous analgosedation, treatment with indomethacin or repeated doses of ibuprofen for symptomatic PDA, periventricular leukomalacia, or intraventricular hemorrhage grade 2 or more, assessed by serial cranial ultrasound. The study was approved by the ethics committee of Innsbruck Medical University (study no. UN3019).

\section{Sedation}

Infants in need of surfactant treatment in the delivery room received bolus analgosedation, which was at the discretion of the attending neonatologist (benzodiazepines, opioid derivates, ketamine, and opioid antagonist).

\section{Diagnosis and Treatment of PDA}

All preterm infants were routinely screened by echocardiography within 48-72 h of PNA. If a left-to-right shunt over the ductus arteriosus was diagnosed, pharmacological treatment with ibuprofen (Pedea; Orphan Europe SARL, Puteaux, France) was started according to the following method: initial dose of $10 \mathrm{mg} / \mathrm{kg}$ and second and third doses of $5 \mathrm{mg} /$ $\mathrm{kg}$ ibuprofen intravenously administered every $24 \mathrm{~h}$. On day 6 of life, echocardiography was performed for the re-evaluation of the PDA.

\section{Clinical Characteristics of the Study Cohort}

In total, 73 children were eligible for the study. Eight infants were excluded, including one infant who developed periventricular leukomalacia, two infants who were treated with indomethacin, and five infants who received a second cycle of ibuprofen treatment. Another four infants had a PDA on routine echocardiography but did not receive postnatal analgosedation. They were post hoc excluded from further analysis due to the limited number of infants within this study group. The final cohort comprised 61 infants, who were included in 
the following three subgroups: (i) reference group: received no analgosedation and had no PDA $(n=21)$, (ii) sedation group: received analgosedation but did not have PDA $(n=20)$, and (iii) PDA group: received analgosedation and one course of ibuprofen treatment for PDA $(n=20)$. Perinatal characteristics of the cohort in relation to the study groups are given in Table 2 .

\section{aEEG Recording}

Two-channel aEEG was recorded with the BrainZ Instruments BRM2/3 monitor (BrainZ Instruments; Natus Medical, San Carlos, CA) using hydrogel electrodes with standard electrode placement at $\mathrm{C} 3, \mathrm{P} 3, \mathrm{C} 4$, and $\mathrm{P} 4$ regions according to the 10-20 International System of Electrode placement. This tool uses the parietal (P3, P4) electrodes for cross-cerebral aEEG recordings. Recording quality was monitored by continuous measurement of the electrode impedance. Traces with impedance $>5 \mathrm{kOhm}$, or with obvious or marked artifacts, were excluded from the analysis. aEEG was commenced no later than $6 \mathrm{~h}$ after birth and continued for a total length of $72 \mathrm{~h}$. We analyzed six defined representative time periods each with a duration of $6 \mathrm{~h}$ : age $6-12 \mathrm{~h}(n=52$ recordings $), 18-24 \mathrm{~h}(n=53)$, $30-36 \mathrm{~h}(n=50), 42-48 \mathrm{~h}(n=46), 54-60 \mathrm{~h}(n=46)$, and 66-72 $\mathrm{h}(n$ $=45$ ). We chose this approach in order to reach for the evaluationfeasible, representative number of tracings in each infant. Serial aEEG recordings were performed during the first 4 wk of life (days $7,14,21$, and $28 ; n=51-57$ ), each for a duration of $4-6 \mathrm{~h}$.

\section{aEEG Assessment}

The 6-h epochs, and the weekly recordings, were evaluated according to three methods:

1. Visual assessment of dominating background activity (visual background) (continuous, discontinuous, burst suppression, low voltage, flat) (12) and onset and presence of cycling defined as FSV and obvious periodic widening (19).

2. Background scoring according to Burdjalov et al., including four single parameters: $\mathrm{CO}, \mathrm{CY}, \mathrm{ALB}, \mathrm{BS}-\mathrm{ALB}$, together with their sum (TS) (11).

3. Amplitude parameters (minimum, mean, and maximum) automatically extracted from the aEEG tracing using the Analyze Research software (Version 1.3; BrainZ Instruments; Natus Medical) (1). aEEG recordings were obtained by two investigators (M.B. and E.G.), who were experienced but had no specific training. The aEEG variables were characterized for the three subgroups of infants and assessed in relation to GA, PNA, and PMA. A synopsis of the assessed aEEG parameters and scores is given in Table 3.

\section{Statistical Analysis}

Data analyses and representations were performed within the statistical environment $R$ (R Development Core Team, 2010) (30). Descriptive data are reported as mean (SD), median, and range of variation for continuous parameters and as proportion (\%) of the whole population for categorical outcomes. Potential bias in the experimental design was assessed with the two-tailed Welch $t$-test, the Wilcoxon test, and the one-tailed Barnard test (31). Proportional hazards modeling was applied to evaluate the impact of study group and GA on time to FSV and first obvious widening. Modeling of the time to FSV was approached by negative binomial regression. Burdjalov scores and the visual background assessment were compared using generalized estimating equations, and distributions of the amplitude parameters were compared by generalized least square regression. Models were independently built from the data obtained in the first $(6-72 \mathrm{~h}$ ) and the second (week $1-4)$ monitoring phases. Initial model specification strictly follows the experimental design, comprising GA, PNA, and PMA (accommodating linear and quadratic relationships with time), and study group terms as main effects and an interaction term between study group and age to examine the time-dependent changes specific to a particular study group. For generalized estimating equation models, intraindividual correlation was addressed using an autoregressive covariance structure in the first monitoring period $(6-72 \mathrm{~h})$ and an exchangeable correlation structure in the second period (week 1-4). Several specifications of the generalized least square regression error term were allowed in order to include the longitudinal structure and deal with potential time-dependent heteroscedasticity. Model selection and diagnostics follow the protocols of Zuur et al. (32). Incidences evaluated in a single aEEG session (i.e., corresponding to a given PNA) were calculated with Firth's penalized-likelihood logistic regression (33). Estimated parameters are accompanied by their $95 \%$ confidence intervals.

\section{STATEMENT OF FINANCIAL SUPPORT}

This study was supported by the European Commission (Sixth Framework Programme, contract LSHM-CT-2006-036534) and by a research grant (no. 1275) from the Jubilee Funds of the Austrian National Bank. No form of payment was given to anyone to produce the manuscript.

Disclosure: The authors declared no conflict of interest.

\section{REFERENCES}

1. Bowen JR, Paradisis M, Shah D. Decreased aEEG continuity and baseline variability in the first 48 hours of life associated with poor short-term outcome in neonates born before 29 weeks gestation. Pediatr Res 2010;67: $538-44$.

2. El-Dib M, Massaro AN, Glass P, et al. Early amplitude integrated electroencephalography and outcome of very low birth weight infants. Pediatr Int 2011;53:315-21.

3. Hellström-Westas L, Rosén I. Electroencephalography and brain damage in preterm infants. Early Hum Dev 2005;81:255-61.

4. Kato T, Okumura A, Hayakawa F, Tsuji T, Natsume J, Hayakawa M. Amplitude-integrated electroencephalographyin preterminfantswith cystic periventricular leukomalacia. Early Hum Dev 2011;87:217-21.

5. Klebermass K, Olischar M, Waldhoer T, Fuiko R, Pollak A, Weninger M. Amplitude-integrated EEG pattern predicts further outcome in preterm infants. Pediatr Res 2011;70:102-8.

6. Niemarkt HJ, Andriessen P, Peters $\mathrm{CH}$, et al. Quantitative analysis of amplitude-integrated electroencephalogram patterns in stable preterm infants, with normal neurological development at one year. Neonatology 2010;97:175-82.

7. Olischar M, Klebermass K, Kuhle S, et al. Reference values for amplitudeintegrated electroencephalographic activity in preterm infants younger than 30 weeks' gestational age. Pediatrics 2004;113(1 Pt 1):e61-6.

8. Thoresen M, Hellström-Westas L, Liu X, de Vries LS. Effect of hypothermia on amplitude-integrated electroencephalogram in infants with asphyxia. Pediatrics 2010;126:e131-9.

9. Toet MC, van Rooij LG, de Vries LS. The use of amplitude integrated electroencephalography for assessing neonatal neurologic injury. Clin Perinatol 2008;35:665-78, v.

10. Shankaran S, Pappas A, McDonald SA, et al.; Eunice Kennedy Shriver National Institute of Child Health and Human Development Neonatal Research Network. Predictive value of an early amplitude integrated electroencephalogram and neurologic examination. Pediatrics 2011;128:e112-20.

11. Burdjalov VF, Baumgart S, Spitzer AR. Cerebral function monitoring: a new scoring system for the evaluation of brain maturation in neonates. Pediatrics 2003;112:855-61.

12. Hellström-Westas L, Rosén I, de Vries LS, et al. Amplitude-integrated EEG classification and interpretation in preterm and term infants. NeoReviews 2006;7:76-87.

13. Kato T, Okumura A, Hayakawa F, Tsuji T, Natsume J, Watanabe K. Evaluation of brain maturation in pre-term infants using conventional and amplitude-integrated electroencephalograms. Clin Neurophysiol 2011; 122:1967-72.

14. Klebermass K, Kuhle S, Olischar M, Rücklinger E, Pollak A, Weninger M. Intra- and extrauterine maturation of amplitude-integrated electroencephalographic activity in preterm infants younger than 30 weeks of gestation. Biol Neonate 2006;89:120-5.

15. Zhang D, Liu Y, Hou X, et al. Reference values for amplitude-integrated EEGs in infants from preterm to 3.5 months of age. Pediatrics 2011;127: e1280-7. 
16. Helderman JB, Welch CD, Leng X, O’Shea TM. Sepsis-associated electroencephalographic changes in extremely low gestational age neonates. Early Hum Dev 2010;86:509-13.

17. ter Horst HJ, Jongbloed-Pereboom M, van Eykern LA, Bos AF. Amplitudeintegrated electroencephalographic activity is suppressed in preterm infants with high scores on illness severity. Early Hum Dev 2011;87:385-90.

18. Wikström S, Lundin F, Ley D, et al. Carbon dioxide and glucose affect electrocortical background in extremely preterm infants. Pediatrics 2011;127: e1028-34.

19. Kuint J, Turgeman A, Torjman A, Maayan-Metzger A. Characteristics of amplitude-integrated electroencephalogram in premature infants. J Child Neurol 2007;22:277-81.

20. Sisman J, Campbell DE, Brion LP. Amplitude-integrated EEG in preterm infants: maturation of background pattern and amplitude voltage with postmenstrual age and gestational age. J Perinatol 2005;25:391-6.

21. Soubasi V, Mitsakis K, Nakas CT, et al. The influence of extrauterine life on the aEEG maturation in normal preterm infants. Early Hum Dev 2009;85:761-5.

22. Hagmann CF, Robertson NJ, Azzopardi D. Artifacts on electroencephalograms may influence the amplitude-integrated EEG classification: a qualitative analysis in neonatal encephalopathy. Pediatrics 2006;118:2552-4.

23. Shany E. The influence of phenobarbital overdose on aEEG recording. Eur J Paediatr Neurol 2004;8:323-5.

24. Suk D, Krauss AN, Engel M, Perlman JM. Amplitude-integrated electroencephalography in the NICU: frequent artifacts in premature infants may limit its utility as a monitoring device. Pediatrics 2009;123:e328-32.
25. Supcun S, Kutz P, Pielemeier W, Roll C. Caffeine increases cerebral cortical activity in preterm infants. J Pediatr 2010;156:490-1.

26. van den Berg E, Lemmers PM, Toet MC, Klaessens JH, van Bel F. Effect of the "InSurE" procedure on cerebral oxygenation and electrical brain activity of the preterm infant. Arch Dis Child Fetal Neonatal Ed 2010;95: F53-8.

27. Kurtis PS, Rosenkrantz TS, Zalneraitis EL. Cerebral blood flow and EEG changes in preterm infants with patent ductus arteriosus. Pediatr Neurol 1995;12:114-9.

28. Lemmers PM, Molenschot MC, Evens J, Toet MC, van Bel F. Is cerebral oxygen supply compromised in preterm infants undergoing surgical closure for patent ductus arteriosus? Arch Dis Child Fetal Neonatal Ed 2010;95: F429-34.

29. Limperopoulos C, Bassan H, Kalish LA, et al. Current definitions of hypotension do not predict abnormal cranial ultrasound findings in preterm infants. Pediatrics 2007;120:966-77.

30. R Development Core Team (2010). R: A Language and Environment for Statistical Computing. Vienna, Austria: R Foundation for Statistical Computing. ISBN 3-900051-07-0, (http://www.R-project.org).

31. Barnard GA. Significance tests for 2 X 2 tables. Biometrika 1947;34: 123-38.

32. Zuur AF, Ieno EN, Walker N, et al. Mixed Effects Models and Extensions in Ecology With R, 1st edn. New York, USA: Springer Science+Business Media, 2009:574.

33. Heinze G. A comparative investigation of methods for logistic regression with separated or nearly separated data. Stat Med 2006;25:4216-26. 